Proceedings of the 2011 Winter Simulation Conference

S. Jain, R.R. Creasey, J. Himmelspach, K.P. White, and M. Fu, eds.

\title{
IMPLEMENTATION OF A SIMULATION-BASED SHORT-TERM LOT ARRIVAL FORECAST IN A MATURE 200MM SEMICONDUCTOR FAB
}

\author{
Wolfgang Scholl \\ Infineon Technologies Dresden \\ Koenigsbruecker Strasse 180 \\ Dresden 01099, GERMANY \\ Daniel Noack \\ Oliver Rose \\ Dresden University of Technology \\ Institute of Applied Computer Science \\ Dresden 01062, GERMANY
}

\author{
Boon Ping Gan \\ Peter Lendermann \\ D-SIMLAB Technologies Pte Ltd \\ 8 Jurong Town Hall Road \#30-04 JTC Summit \\ Singapore 609434, SINGAPORE \\ Patrick Preuss \\ Falk Stefan Pappert \\ D-SIMLAB Technologies GmbH \\ Wiener Platz 6, \\ Dresden 01069, GERMANY
}

\begin{abstract}
The ability to perform lot arrival forecast at work center level is a key requirement for pro-active FAB operation management. Visibility to this information enables preemptive resource allocation and bottleneck management. Today, the work center lot arrival forecast is achieved through the use of short term simulation technique in Infineon Dresden. High fidelity simulation model that includes detailed modeling feature such as attribute-based sampling procedure, dedication and temporary tool blocking is built automatically through the transformation of data queries from data sources. In this paper, we present the results of our model validation work, comparing the FAB and forecasted lot arrival of the defect density measurement work center. Due to the high capacity demand of automotive product that requires more than 20 inspection steps; engineering lots and preventive maintenance of DDM must be scheduled at the right time. This can only be achieved with high quality lot arrival forecast.
\end{abstract}

\section{INTRODUCTION}

Within the last months at Infineon Technologies Dresden, a discrete event simulation based short-term forecast system, D-SIMForecaster, was deployed. Originally, simulation has been used for mid-term forecast of fab performance indicators in addition to static capacity planning. Up to now, important operational decisions in the production line, like definition of the optimum date for a preventive maintenance, had to be done without consideration of the expected WIP situation. At the end, these activities were typically scheduled for the last date of maturity with the motivation of cost reduction. Sometimes this procedure resulted in huge capacity problems, like shown in the example of work center CVD_1 (Figure 1). Based on a lot arrival prediction, here the preventive maintenance had been started two days earlier, that means on the first due date within the maturity window. This situation could have been avoided if a short term simulation is already conducted prior to the execution of the preventive maintenance activity. Today, our focus is to realize a short term simulation solution with forecast accuracy of 1 to 3 days (at most 7 days), which is a typical response time required for operational decisions. Some related work on short term simulation (also known as online simulation) can be found in Bagchi et al. (2008). 


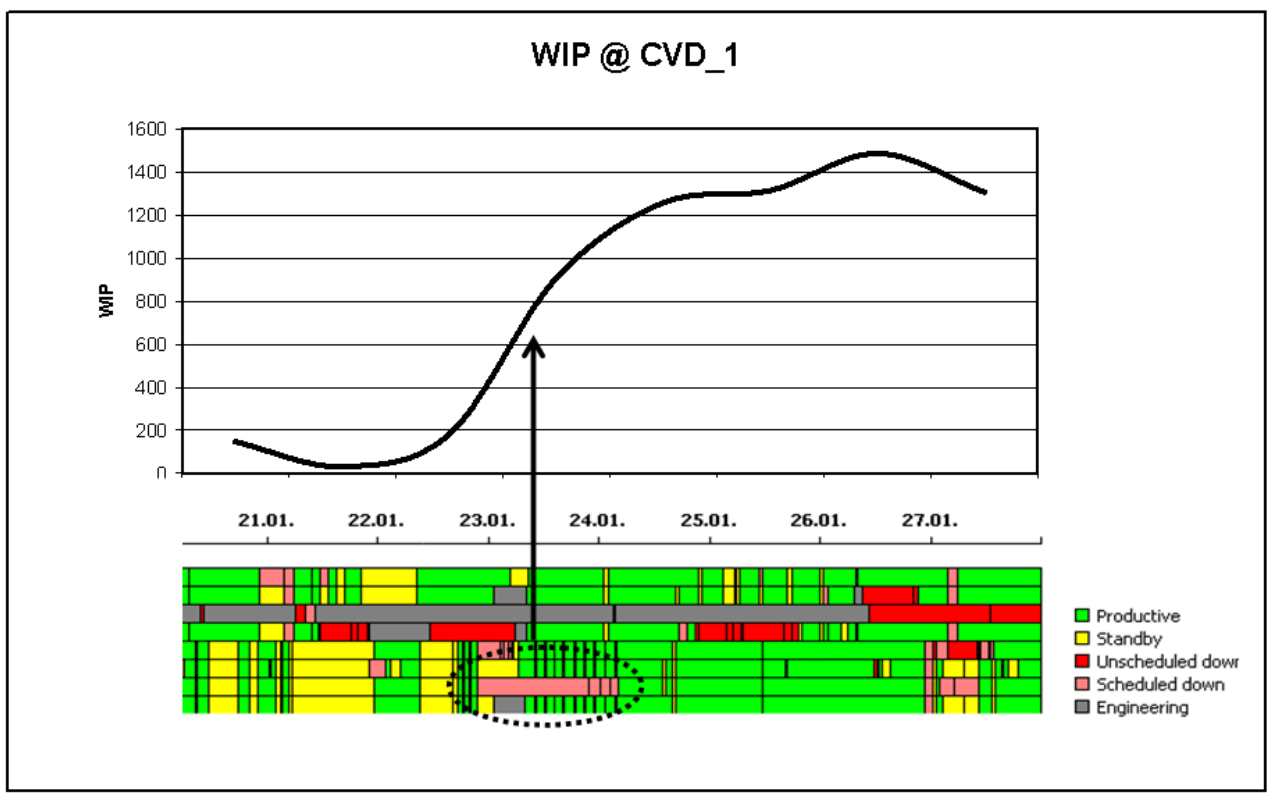

Figure 1: WIP and tool status CVD_1

In addition to the CVD work center that we discussed, another critical production area calling for a short-term lot arrival forecast, is wafer inspection. At Infineon Dresden the automotive products have to be processed with high reliability requirements. To ensure the quality, more than 20 to 30 inspection steps on expensive, highly utilized defect density measurement tools are necessary. Also, other products run on this work center with wafer-start dependent sampling rates. In addition to these, special measurements with volatile lot arrivals have to be processed, particularly in case of time-critical tool re-qualifications after maintenance or process problems in the line. The stochastic overlapping of these material flows has often induced temporary capacity problems, which in turn similarly caused cycle time violations of critical products deliveries (as illustrated in Figure 2). With the ability to forecast WIP trend at this work center, we can avoid work center load peaks through better scheduling of special measurements and, of course, engineering and maintenance activities or the proactive temporary adjustment of sampling rates for less critical process steps. This triggers the need to model sampling in higher granularity for this work center. We need to be able to differentiate between highly critical measurement and less critical measurement operations. This is achieved through attribute based sampling, where lots are marked with different attributes and attribute values to represent the need of performing a series of measurement operations.

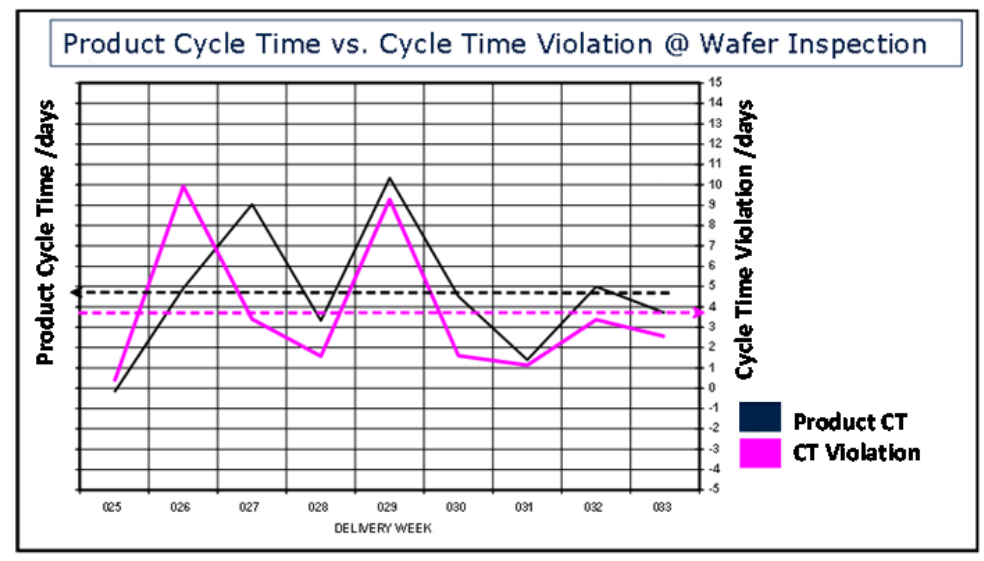

Figure 2: Product cycle time deviation in dependence on cycle time violation of wafer inspection 
Beside enhancing the granularity of sampling, we also focused on realistic modeling of the lithography area. Some important modeling features such as lot size dependent throughput and setup-optimized dispatching were incorporated in our earlier work (Scholl et al. 2010). But we realized that this was not sufficient to realize good forecast accuracy for lithography area as the forecast results were overly optimistic. One crucial modeling feature that we have identified is the need to further enhance the dedications modeling. They are tool specific process releases ("white list dedications") and temporary tool blocking ("black list dedications"). In lithography area temporary blocking information can be relevant for specific classes of processes (e.g., in case of resist problems) or hardware based (e.g., in case of optical problems).

One main requirement on a short-term simulation is the ability to instantiate the simulation model based on current Fab status within minutes. This can only be achieved through a fully automatic model building procedure in connection with a not too extensive modeling approach. That means, there is no need to have a one-to-one copy of machines with all hardware components, or dispatching rules with all details from reality. It is sufficient to model the typical behavior, e.g., lot size dependent processing time or the effect of typical logistical constraints (e.g., time windows between process steps). The modeling granularity also depends on the basic data availability from Infineon IT data sources. Some data that are not available such as tool down statistics, send ahead rates in lithography and CMP area, and transport times were derived from real historical data. Automatic material handling system is currently not modeled, but will be included in a future project in connection with an extended range of application of shortterm simulation. To achieve real-time simulation model instantiation, we adopted an intelligent data management approach. Basic data are classified in a hierarchy with different update frequency (from daily, weekly to monthly) reducing the daily data load time to a couple of minutes. A data correction module is also introduced to correct missing or inconsistent data to avoid permanent interruption of the simulation execution. Error reporting and a user-friendly graphical user interface solution is provided for continuous model improvement. Some works related to automatic simulation model generation can be found in Son and Wysk (2001).

In this paper, we discussed the validation results of two selected pilot work centers: (1) wafer inspection, and (2) a selected CVD tool. Initial focus on model validation was to resolve any model initialization issues, preventing the propagation of errors to subsequent days of simulation. We have identified that the initial discrepancy were due to basic data inconsistencies, unscheduled down events that deviate from reality, lot release plans that are only available for 7 days weekly, and storage lot plan releases that are not available at all. But the discrepancy were adjusted through several simplification in modeling, such as lot release plans is replicated for time period that is not available. We achieved close to reality model initialization, good daily forecast accuracy with the simulation model that was built automatically within minutes.

\section{D-SIMFORECASTER}

Based on the requirements for short term simulation we developed a software system which enables a user to utilize short term simulation in a semiconductor environment. Different modules address the challenges posed by short term simulation to collectively generate a simulation model from the running production system. A detailed overview on the system is shown in Figure 3.

In general, D-SIMForecaster is made up of three major software components: (1) A component that queries production databases for master, snapshot, and historical data, and processes the data into a consistent dataset, (2) A component that creates and manages (simulation) scenarios from this consistent dataset, and (3) A component that exports the scenario into a file format that the simulation engine can understand, and import reports from the simulation run to be shown to user. Referring to Figure 1, the first component is made up of Data Model Query Engine (DMQE), Historical Data Analyzer (HDA), and Data Post Processing (DPP). The second component comprises of Simulation Model Query Engine (SMQE) and Model Configuration Graphical User Interface (MCGUI). Lastly, the third component comprises of Model Scenario Generation Engine (MSGE) and Intelligent Reporting Engine (IRE). The system is created in a modularized fashion to allow flexibility of implementing the solution to different Fabs. For exam- 
ple, if a Fab has a different production databases, we need to replace only the DMQE and HDA. Likewise, when we need to use a different simulation engine, only MSGE and IRE need to be replaced. Using this system architecture, we allow maximum flexibility with plug-and-play of software components. In the following sections we shall give a short introduction on the different modules, their core functionality and approaches used to satisfy requirements of short term simulation. Discussion on issues related to the design of the data model can be found in Noack et al. (2010).

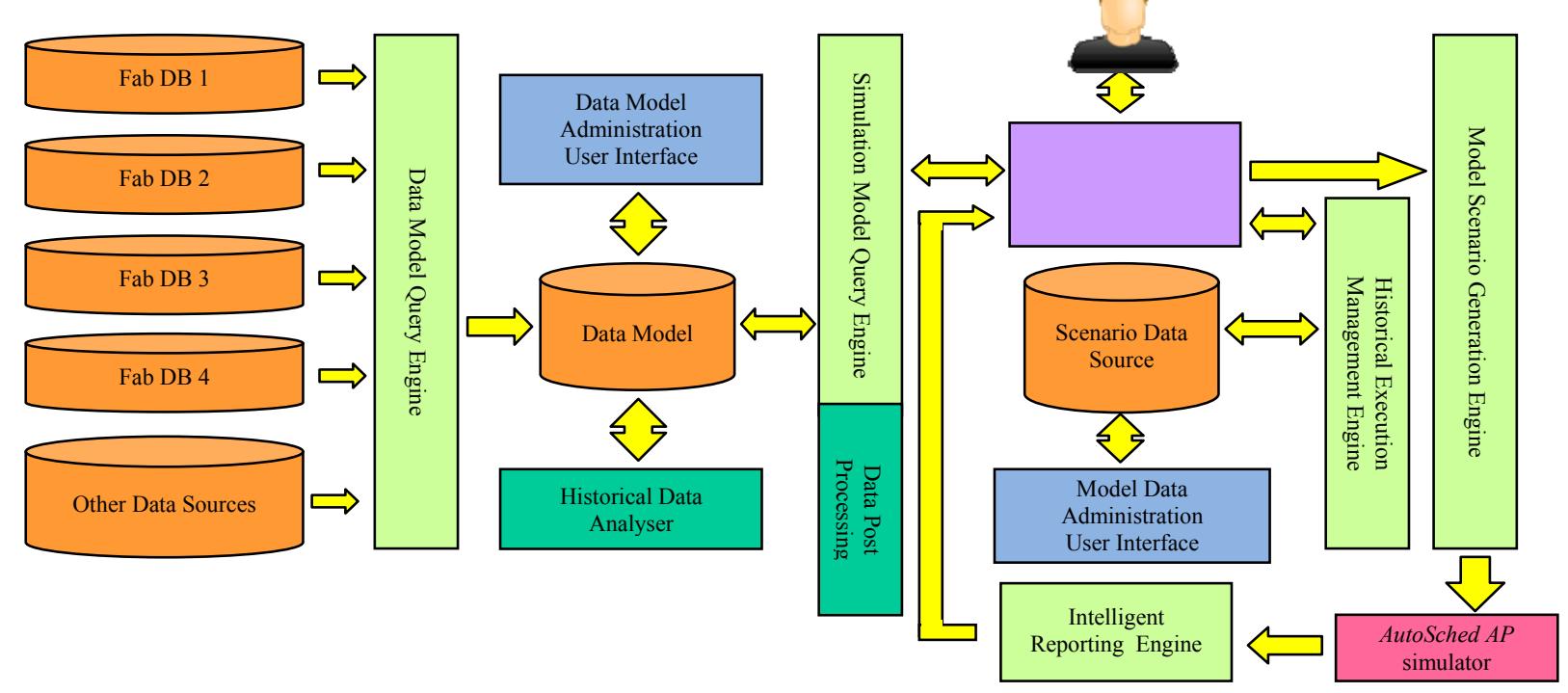

Figure 3: D-SIMForecaster system architecture

\subsection{Key Components}

The Data Model Query Engine (DMQE) is used to collect input data from the Infineon data sources which are required to build up a short-term simulation model. As all these data have a different change frequency they are classified into groups based on their update frequency and order of usage as summarized in Table 1. Master data such as process routes, equipment set, process time and throughput form a large part of the data required for the building of a simulation model. But one key characteristic of master data is also that it does not change often. As such, master data is updated only once a week, and this significantly reduces the data query volume and time for instantiation of simulation models. The lead time between a simulation model instantiation is started until a simulation model is built must be fast (in the range of minutes). Otherwise, the simulation model might be already outdated before it can be used for the purpose of short term simulation.

Snapshot data such as work-in-progress (WIP), lot releases plan, temporary dedication, and preventive maintenance plan change with a much shorter frequency. They represent the dynamism of the Fab production line. This data is only queried when a simulation model instantiation is initiated. As the data volume is much smaller, the speed of query is fast and does not form a bottleneck to data query speed.

As not all data are available from production databases, some data such as sampling rate, rework rate, lot split rate, hold rate and duration, mean-time-to-failure (MTTF), and mean-time-to-repair (MTTR) must be calculated. This calculation is performed through analysis of the historical lot traces and equipment state traces using our Historical Data Analyzer (HDA). To ensure that the data calculated is of high statistical confidence, we took the historical traces for the past 60 days into consideration. We are not taking historical traces that are too far into the past as those data will be of less relevance due to the dynamism of a wafer fabrication line. In addition, our HDA also avoids calculating the values based on a short-term abnormality with Fab behavior, such as exceptionally long down time for an equipment for the time period of trace. This is achieved through comparing the average values computed in the past versus 
the average values computed with the historical traces of current time period. If the deviation is longer than a pre-defined value, user will be alerted of this abnormality and proper statistical analysis can be conducted to derive the right value.

Table 1: Data Classification

\begin{tabular}{|l|l|l|}
\hline Data Class & Description & $\begin{array}{l}\text { Update } \\
\text { Frequency }\end{array}$ \\
\hline Master & $\begin{array}{l}\text { Static base data of the Fab such as route, equipment set, process } \\
\text { time and throughput. }\end{array}$ & Weekly \\
\hline Snapshot & $\begin{array}{l}\text { Dynamic Fab data such as work-in-progress (WIP), temporary } \\
\text { dedication, preventive maintenance plan, etc. }\end{array}$ & Daily \\
\hline Trace & $\begin{array}{l}\text { Historical real Fab data which is used to calculate derived data } \\
\text { such as sampling rate, rework rate, lot split rate, mean-time-to- } \\
\text { failure, mean-time-to repair. }\end{array}$ & Weekly \\
\hline $\begin{array}{l}\text { Manually } \\
\text { Maintained }\end{array}$ & $\begin{array}{l}\text { Small set of information that is not available from any data } \\
\text { sources such as setup time, equipment model classification, etc. }\end{array}$ & On demand \\
\hline
\end{tabular}

Some data are manually maintained as they are not available from any of the production databases, and are also not feasible to be calculated/derived automatically. We kept this set of data as minimal as possible to avoid frequent manual intervention by the user . Using this approach, we ensure that the simulation model can be built automatically at least $98 \%$ of the time, and forecast can be conducted daily without any major issues.

As data from production databases can never be perfect, we developed a Data Post Processing (DPP) module that is responsible to correct any data inconsistency. Data exceptions can happen as the data is gathered from various data sources and formats, i.e., SQL databases, Excel documents or Access files, where different naming conventions or levels of granularity are used. DPP attempts to automatically correct the data issues by using one of the predefined solutions. One example is that an operation of a WIP lot cannot be found in the process route. DPP resolves this issue by moving the operation of the WIP lot to the next operation within the process route. Another example is that the process time or throughput data is not available for a particular process at an equipment. DPP resolves this issue by taking an average of the process time and throughput of similar processes (identified by the name with some prefix). We introduced the DPP module to ensure that simulation model can be generated automatically with the least user intervention. As discussed earlier, we are aiming to achieve successful automatic model generations and forecast run $98 \%$ of the time. In the event that no solution can be found by DPP, the data issue is marked as unresolved and presented to the user via the Model Configuration Graphical User Interface (MCGUI) for correction.

The Simulation Model Query Engine (SMQE) is a model transformation module which combines data of both snapshot and master into a single representation of the Fab, i.e., the Fab simulation model, at the time the snapshot is taken. During this transformation, irrelevant Fab information is removed to create a lean data set which reduces the amount of simulation objects thereby enhancing simulation speed. The data associated with the simulation model is recorded into the Scenario Database. The recording of instantiated simulation model facilitates the user to perform simulation experiments on current or past snapshots. The functionality of retrieval of current and past snapshots is provided through a scenario management system, Historic Execution Management Engine (HEME). Working hand in hand with the MCGUI it is possible to change scenarios to analyze the effects of modified Fab settings.

In order to simulate an instantiated simulation model, we need a simulation engine. In this work, we use AutoSched $A P(A S A P)$ simulator for this purpose. The Model Scenario Generation Engine (MSGE) exports the simulation model to a file format that is understandable by the $A S A P$ simulator, as illustrated in Figure 4. Upon exporting the files, $A S A P$ is triggered to run the simulation for a daily forecast. Reports 
generated by $A S A P$ are read by the Intelligent Reporting Engine (IRE), and converted to charts and reports that are required by the user. Some examples of charts are: daily WIP, moves, wafers arrival and depature at work center level, as illustrated in Figure 5.

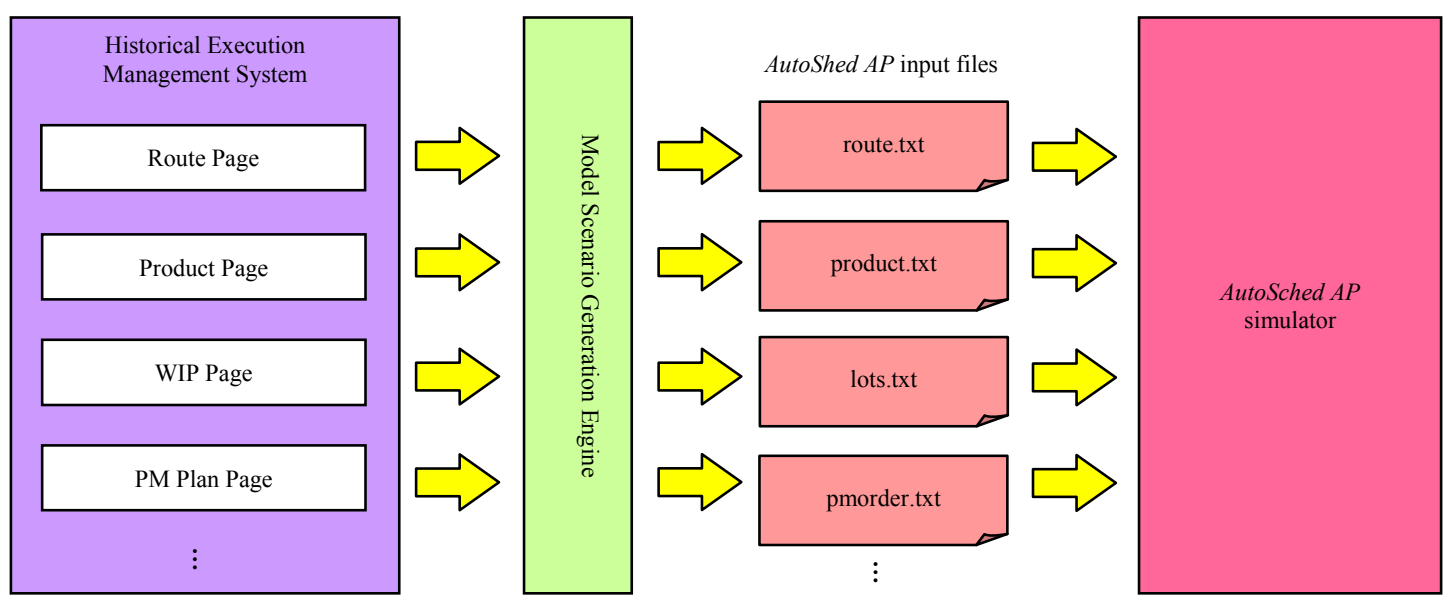

Figure 4: An Illustration of MSGE

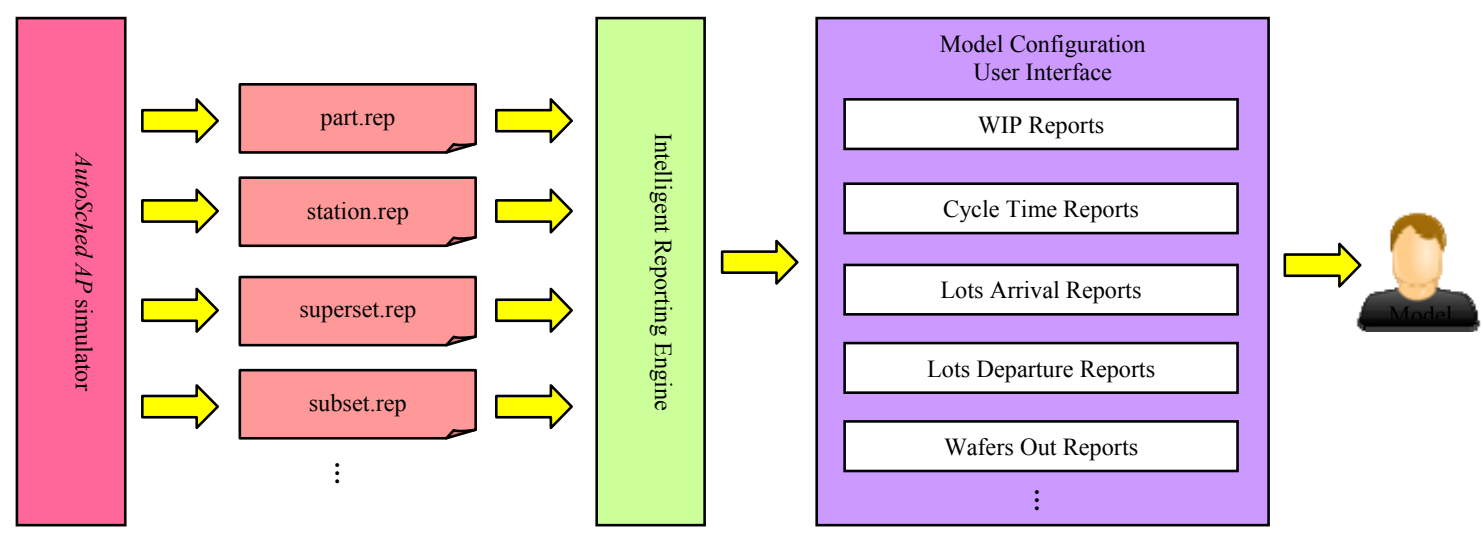

Figure 5: An Illustration of IRE

\subsection{System Performance}

Two of the key challenges for short term simulation are the need for high granularity simulation model, and at the same time, a real time capability to instantiate this simulation model. The reason for this real time need is that the system is used for operational support, and information must be made available to line manager as early as possible. This enables the line managers to respond to the potential Fab problems quickly. Furthermore the instantiated model is losing accuracy and is no longer a good representation of current Fab status if the time taken to build the model is long (a delay of more than 15 minutes is considered as not feasible anymore). Therefore we are faced with challenging time restrictions to ensure that the system can provide decision support promptly before the result of the calculations are obsolete. Using techniques discussed in Section 2.1, we were able to reduce the creation time of a simulation model of the whole Fab to less than 10 minutes. Figure 6 gives an idea of the total time required from the time of model instantiation until the simulation run is completed. As can be seen, the simulation model is created within 6 minutes, with the efficiently implemented data correction and data transformation modules. With this, there is almost no time for the original system to deviate by a large extent from the snapshot used to run the simulation, thus keeping the simulation on track and producing relevant forecasts. 


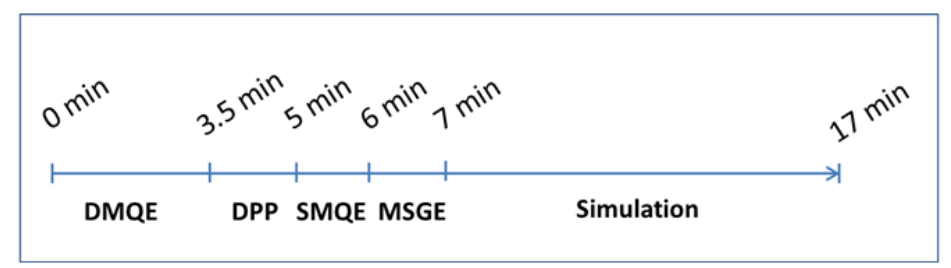

Figure 6: Timeline of a Fab snapshot

\section{MODELING FIDELITY}

\subsection{Overview}

In order to provide a good work center level daily forecast with a time horizon of 7 days, the short term simulation model was built with good fidelity as described in our earlier work (Scholl et al. 2010). Modeling elements included are: new lot release plan, storage lot release strategy, lot size dependent process time and throughput (with five classes of equipment model), process route, equipment dedication (mapping of processes to specific equipment set), preventive maintenance schedule, and sampling process. In addition, the simulation model was initialized with the latest Fab state, which includes the WIP situation and equipment state. Incorporating these modeling elements gave us good work center forecast accuracy in WIPs, wafers arrival and departure.

Even though we have achieved good forecast accuracy for many work centers, we did not manage to get sufficiently good forecast accuracy for the measurement work centers. One particular important measurement operation is defect density, which was modeled as a constant delay and throughput in our earlier work. Today, this is no longer sufficient as a high percentage of our WIP see this operation, and the work centers related to this operation have become some of the Fab bottlenecks. To address this new challenge, we extended our simulation model to consider the defect density operations and related work centers with high fidelity. This new modeling feature is termed as attribute based sampling. Detailed discussion follows in the next section.

We have also seen forecast accuracy issue related to the lithography work centers. The primary reason for this is the dynamic process dedication characteristics of the lithography related work centers. Dedication is not only determined by the operation process that a lot needs, it is also determined by the lot process route and product. Adding on to this complexity, some of the lithography equipment are temporarily blocked from some process routes or products from time to time. Without taking these constraints into consideration, our simulation model is more optimistic (in terms of throughput) than reality. To address this issue, we increased the fidelity of dedication modeling for lithography work centers.

\subsection{Attribute Based Sampling}

Each lot in the Fab is tagged with a number of attributes (set with some values) which are used for operational control. Some examples of these lot attributes are dispatch priority, equipment allocation, and sampling. The sampling lot attributes are relevant to the defect density related operations. The lot attributes determine if the lot should go through the defect density measurement operations, and at what sampling rate. Typically, the setting of the attributes is product dependent. In addition, the sampling lot attributes are set at various point in time during the manufacturing operations. Some are set at the beginning of the lot releases, while some are set a few operations before reaching the measurement operations, depending on the Fab conditions or situations. Lot attributes with the latter characteristics are not feasible to be modeled due to the complexity of modeling the criteria/conditions used to set the attributes. Taking these factors into consideration, we adopted the following steps to incorporate the lot attributes relevant for sampling:

1. Identify sampling related lot attributes for defect density. 
2. Determine the set of sampling attributes that is feasible to be modeled.

3. Identify route and operations where these attributes take effect.

4. Historical lot trace analysis to generate the sampling rate for each lot attribute values.

5. Extend the operation sampling rates with attribute dependent operation sampling rates.

6. Initialize the Fab with attribute values for each lot.

Step 1 and 2 were conducted through interview with specialist of the defect density measurement operations. Two lot attributes were identified during this exercise, considering their criticality - number of operations that use this attribute and number of WIP lots with this attribute set. The first attribute is set prior to lot releases to production, and is available in the new lot release plan. The second attribute is set several operations after the lot is released. In the former case, we extended our automatic model generation module to read the associated attributes from the new lot release plan. For the latter case, we gathered the products where the attribute must be set, and set the attribute for all lots belonging to these products. The attribute value stays from the beginning of lot releases until the lot completes all its operations.

In step 3, we identify the operations (for all routes) where the two attributes are relevant through the prefix of the operation process name. Following that, we computed the sampling rate for both lot attributes using 60 days of historical lot traces in step 4. The computed sampling rate varies for different routeoperation combination, and varies for different attribute-value combination. Table 2 below shows an example of the computed sampling rate for route R1, operation 389 and 878, and route R2, operation 385, where attribute ATTR1 could take the value of X, Y, or none. Using the example in row number 1, the operation 389 for route $\mathrm{R} 1$ is executed for 97 lots of a hundred lots (with attribute ATTR1 set to X). The sampling rate value is updated weekly by our Historical Data Analyzer module to ensure that the sampling rate stays relevant.

Table 2: An Example of Attribute Based Sampling Rate

\begin{tabular}{|l|l|l|l|l|}
\hline No. & ROUTE & OPERATION & ATTR1 & SAMPLING RATE \\
\hline 1 & R1 & 389 & X & 0.97 \\
\hline 2 & R1 & 389 & Y & 0.81 \\
\hline 3 & R1 & 389 & & 0.02 \\
\hline 4 & R1 & 878 & X & 0.86 \\
\hline 5 & R1 & 878 & Y & 0.65 \\
\hline 6 & R1 & 878 & & 0.00 \\
\hline 7 & R2 & 385 & X & 1.00 \\
\hline 8 & R2 & 385 & Y & 1.00 \\
\hline 9 & R2 & 385 & & 0.00 \\
\hline
\end{tabular}

In step 5, we extended our simulation model to use this sampling rate. When a lot arrives at an operation, a table look-up is performed to check if the route-operation combination exists in this table. If an entry can be found, the sampling rate is applied depending on the value set for the attribute. If an entry is not found, the step percent of the route-operation pair is used. Step percent is defined as the probability that the operation is executed. It is primarily used to model sampling operations that has no association with attribute-based sampling. With the modeling of attribute based sampling, all WIP lots were also warm started with lot attributes. This is to ensure that lot attributes are used not only for new lot releases, but also for lots that are already in the manufacturing process when the model is created.

\subsection{Lithography High Fidelity Dedication Modeling}

To increase the fidelity of dedication modeling for lithography work centers, we incorporated two new filtering rules into our simulation model. These rules are: enabling list filter (ELF) rule and blocking list filter (BLF) rule. ELF rule is executed to filter out lots that do not meet the filtering criteria, while BLF rule is executed to filter out lots that meet the filtering criteria. The BLF rule is to model the temporary block- 
ing of dedication, which changes dynamically over time and takes precedence over the ELF rule. The filtering criteria of ELF and BLF is a combination of route, product, and operation process names.

\section{EXPERIMENTAL RESULTS}

The model validation exercise was conducted on simulation model that was automatically generated using $D$-SIMForecaster. For the purpose of this paper, we presented the validation results for a snapshot that was taken on $28^{\text {th }}$ May 2011.

\subsection{Model Initialization Validation}

A prerequisite for a good daily forecast accuracy is to have the ability to capture and represent the current Fab state accurately in the simulation model. Figure 7 shows an overview of WIP level comparison between reality and simulation. As you can see, we managed to initialize our simulation model with WIP at the right place (work centers), and at the right time at the beginning of the simulation. There are still minor discrepancies between the Fab state and simulation state due to two key reasons. Firstly, some operations of the route cannot be found in the master data as the production database is not brought up-to-date. Instead of disabling simulation run when this occurs, our automatic data correction module (DPP) updated the WIP lot to the next operation of the route. Secondly, process time variance between reality and simulation result in some lots completing their operations earlier in the simulation. These lots will arrive at the next operation at the beginning of the simulation. These two factors contributed to less than $2 \%$ deviation between reality and simulation, and are not a major hurdle towards forecast accuracy as can be seen in the next section as we present the work center level daily forecast results.



Figure 7: Work centers WIP level at model initialization

\subsection{Defect Density Work Center Forecast}

Figure 8 shows the WIP and moves forecast, with a time horizon of 5 days, for three defect density work centers. As can be seen, our short term simulation model is able to forecast the WIP trend for DD_1 and DD_2 with good accuracy. The increase in WIP for DD_3 was not captured by our simulation due to the lower moves as observed in the moves graph. Nevertheless, DD_2 is the more crucial defect density work 
center due to its high loading, and we managed to achieve a good forecast accuracy. Having this capability means that our maintenance specialist can better manage and plan their preventive maintenance activities. At the same time, the line control department can also reduce sampling rate for less critical processes, to free up capacity when the WIP at these work centers is expected to increase to an alarming level. The operation at defect density area can be managed pro-actively now, rather than reactively as in the past.
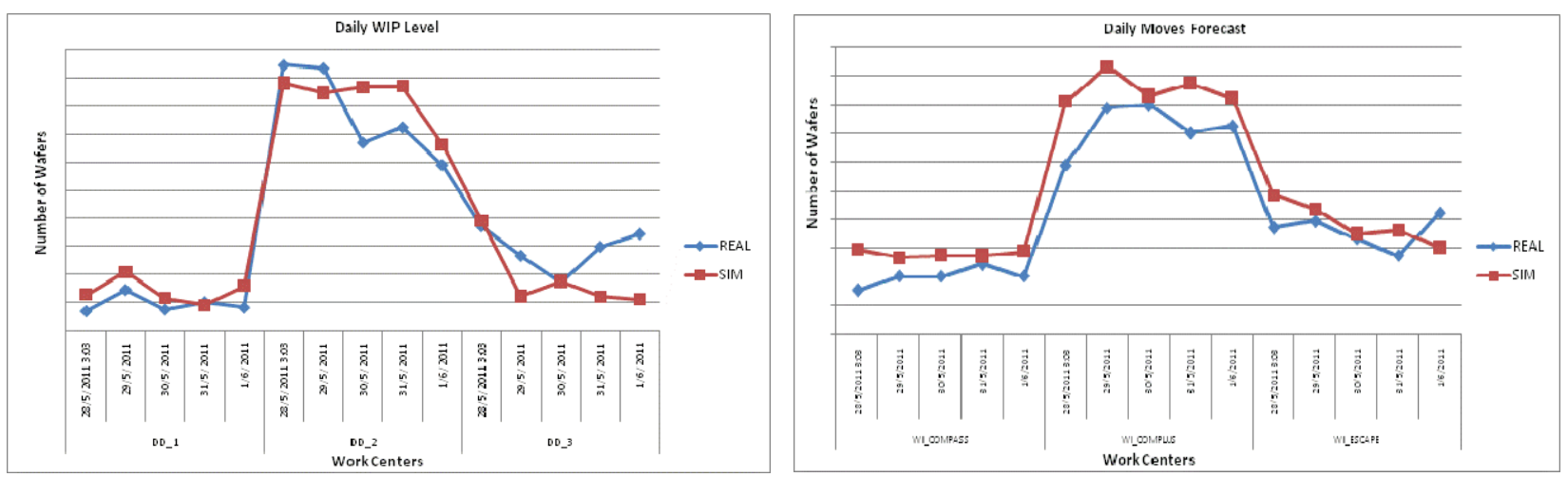

Figure 8: Daily WIP and moves forecast for defect density work centers

\subsection{CVD Work Center Forecast}

As discussed earlier, some of our CVD work centers had been facing WIP waves issue. At times, the preventive maintenance (PM) and engineering activities were scheduled at a time that coincided with WIP waves, resulting in large cycle time variation. It has not been possible to forecast the WIP waves with daily accuracy in the planning of PM and engineering related activities. Figure 9 shows the WIP forecast obtained using our short term simulation model. As can be seen, the WIP trends were forecasted with high accuracy for CVD-1 and CVD-3, but the uptrend on $30^{\text {th }}$ May 2011 for CVD-2 was not forecasted by the simulation. Our analysis has showed that the arrival rate to this work center from upstream work centers (primarily furnaces) is not captured accurately. We are currently working on enhancing the quality for this particular work center as it is one of the most important work centers in our operation, due to its long process time and limited resources availability. Nevertheless, we managed to achieve good forecast accuracy for two other work centers, which can help our maintenance specialist to better plan the PM and engineering related activities. This can definitely reduce the cycle time variability issues that we have been facing due to less than ideal PM planning.

\section{CONCLUSIONS}

In this paper, we presented our deployment of a short term simulation based solution, D-SIMForecaster, for proactive Fab operation management. Snapshot of fab can be taken at any time, and completed within minutes to ensure the forecast is based on the latest fab state. This is essential as a forecast that is based on an outdated fab state is deemed useless. Our approach of segregating data to different classes (based on frequency of change) enables us to achieve fast snapshot taking time. As illustrated earlier, our simulation model is initialized with the fab state, with less than $2 \%$ of deviation from reality. The deviation is primarily contributed by the varying data update frequency. This small deviation is not of significant concern. We are still able to achieve good forecast accuracy as illustrated by the defect density and CVD work centers. Moving forward, we are going to use D-SIMForecaster for wafer out forecast, down to the granularity of product. This will significantly improve the resource planning at our test operations, knowing what products to be expected when. 


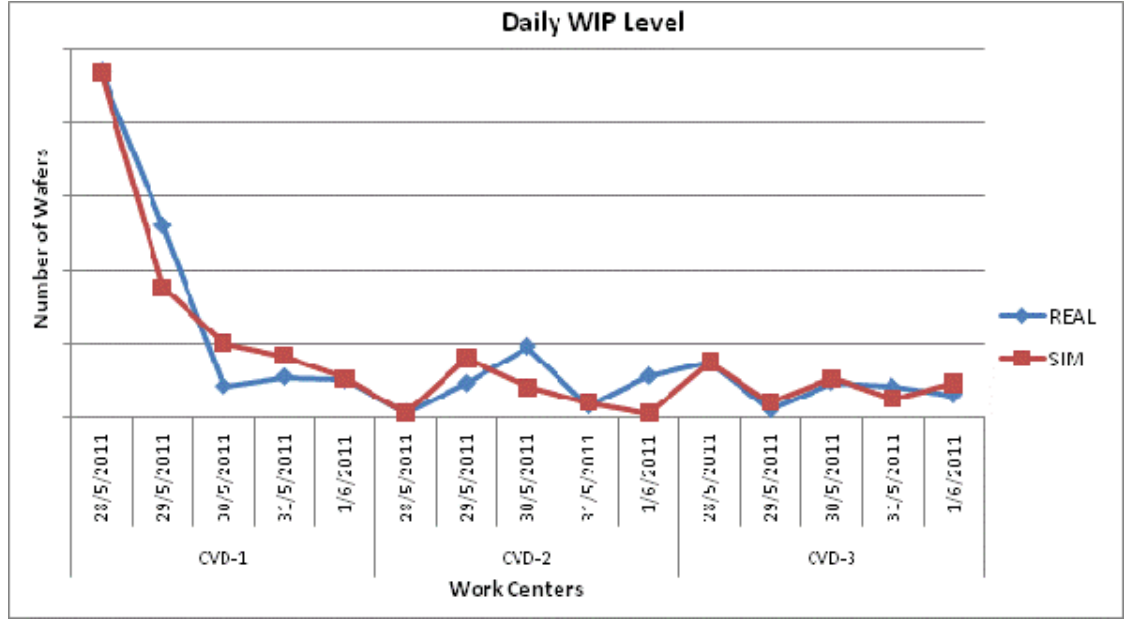

Figure 9: Daily WIP Level Forecast for CVD-1, CVD-2, and CVD-3

\section{ACKNOWLEDGEMENT}

The authors would like to thank Ms Ming Li Peh, Mr Kian Sim Chow, and Mr Pierre Beyer for their contribution towards implementing D-SIMForecaster solution and providing significant support during the model validation exercise.

\section{REFERENCES}

Bagchi, S., C.-H. Chen-Ritzo, S. T. Shikalgar, and M. Toner. 2008. "A Full-Factory Simulator as a Daily Decision-Support Tool for 300mm Wafer Fabrication Productivity." In Proceedings of the 2008 Winter Simulation Conference, edited by S. J. Mason, R. R. Hill, L. Mönch, O. Rose, T. Jefferson, J. W. Fowler, 2021-2029. Piscataway, New Jersey: Institute of Electrical and Electronics Engineers, Inc.

Scholl, W., B.P. Gan, D. Noack, P. Preuss, M.L. Peh, P. Lendermann, and O. Rose. 2010. "Towards Realization of a High-Fidelity Simulation Model for Short-Term Horizon Forecasting in Wafer Fabrication Facilities." In Proceedings of the 2010 Winter Simulation Conference, edited by B. Johansson, S. Jain, J. Montoya-Torres, J. Hugan, and E. Yücesan, 2563-2574. Piscataway, New Jersey: Institute of Electrical and Electronics Engineers, Inc.

Son, Y. J., and R. A. Wysk. 2001. "Automatic Simulation Model Generation for Simulation-Based, RealTime Shop Floor Control." Computers in Industry 45(3):291-308.

Noack D., R. Kohn, M. Mosinski, Z. Zhou, O. Rose, W. Scholl, P. Lendermann, and B. P. Gan. 2010. "Data Modelling for Online Simulation - Requirements and Architecture." In Proceedings of the 2010 FAIM Conference, edited by B. Pulikkal, M. Ramiah, and P. Ronald, 1037-1044.

\section{AUTHOR BIOGRAPHIES}

WOLFGANG SCHOLL works as a Senior Staff Expert for modeling and simulation for Infineon Technologies in Dresden (Germany). He has studied physics at the Technical University of Chemnitz (Germany) and graduated in solid-state physics in 1984. From 1984 to 1995 he worked as a process engineer for ZMD in Dresden. In 1996 he joined Infineon Technologies (former SIMEC) and worked on the field of capacity planning. Since 2003 he is responsible for fab simulation. His email address is wolfgang.scholl@infineon.com.

BOON PING GAN is the CTO of D-SIMLAB Technologies (Singapore). He has been involved in simulation technology application and development since 1995, with primary focus on developing parallel and 
distributed simulation technology for complex systems such as semiconductor manufacturing and aviation spare inventory management. He was also responsible for several operations improvement projects with wafer fabrication clients which concluded with multi-million dollar savings. He is now responsible for managing the technology and product development at D-SIMLAB as well as execution of projects in the semiconductor and engineering domains. He holds a Master of Applied Science degree, specializing in Computer Engineering. His email address is boonping@d-simlab.com.

DANIEL NOACK is a Research Engineer at D-SIMLAB Technologies. His focus is on simulation and simulation-based optimization. He is also a PhD student at Dresden University of Technology. He is a member of the scientific staff of Prof. Dr. Oliver Rose at the Chair of Modeling and Simulation. He received his M.S. degree in computer science from Dresden University of Technology. His email address is daniel@d-simlab.com.

PATRICK PREUSS is a Software Engineer and the Deputy Manager Germany Operations of DSIMLAB Technologies (Germany). He has been working in the development of simulation-based applications for Airbus, German Aerospace Centre and Infineon with focus on heuristic optimization methods since 2005. Patrick holds a M.S. degree in computer science from Dresden University of Technology. His email address is patrick@d-simlab.com.

PETER LENDERMANN is the Co-Founder and CEO of D-SIMLAB Technologies, a Singapore-based company providing simulation-based decision support solutions and services to Aerospace, Semiconductor Manufacturing and other asset-intensive industries. Prior to this he worked at the Singapore Institute of Manufacturing Technology where he led the simulation-related research activities until spinning them off into D-SIMLAB Technologies. He has been engaged in the simulation community since the early 1990's. Peter holds a PhD in Applied High-Energy Physics from Humboldt University in Berlin (Germany) and an MBA in International Economics and Management from SDA Bocconi in Milan (Italy). He is also an Adjunct Associate Professor at the Department of Industrial and Systems Engineering at the National University of Singapore. His email address is peter@d-simlab.com.

OLIVER ROSE holds the Chair for Modeling and Simulation at the Institute of Applied Computer Science of the Dresden University of Technology, Germany. He received an M.S. degree in applied mathematics and a Ph.D. degree in computer science from Würzburg University, Germany. His research focuses on the operational modeling, analysis and material flow control of complex manufacturing facilities, in particular, semiconductor factories. He is a member of IEEE, INFORMS Simulation Society, ASIM and GI. His web address is www.simulation-dresden.com and his email address is oliver.rose@tu-dresden.de.

FALK STEFAN PAPPERT is a Research Engineer at D-SIMLAB Technologies. His focus is on simulation-based scheduling and optimization of production systems. Furthermore he is a $\mathrm{PhD}$ student at Dresden University of Technology as a member of the scientific staff of Prof. Dr. Oliver Rose at the Chair of Modeling and Simulation. He has received his M.S. degree in Computer Science from Dresden University of Technology. He is a member of GI. His email address is falk.pappert@d-simlab.com. 